\title{
Disabling Adverse Event
}

National Cancer Institute

\section{Source}

National Cancer Institute. Disabling Adverse Event. NCI Thesaurus. Code C113380.

An adverse event, and/or its immediate sequelae which is associated with physical or

mental disabilities that affect or limit the ability of a person to perform activities of daily living (eating, ambulation, toileting, etc.). 